\title{
Asthma guidelines: who is guiding whom and where to?
}

Asthma represents a huge clinical load for the health service. Up to 2 million adults (5\%) and 1 million children $(10 \%)$ in the United Kingdom have some degree of asthma. Over 90000 are admitted to hospital each year for acute asthma and about 2000 die, of whom half are under 60 years of age. ${ }^{1}$ As well as the direct costs to the health service of managing asthma, the morbidity causing time off work and absence from school is a substantial but less visible cost both for the individual and for the country. How should the health service respond to this problem?

In 1989-90 Australian, ${ }^{2}$ Canadian, ${ }^{3}$ and British $^{45}$ documents were published that set out guidelines for managing asthma in adults. These were accompanied and followed by paediatric guidelines ${ }^{67}$ and more recently by an American version ${ }^{8}$ and a well referenced international version. ${ }^{9}$ The 1990 British version was unusual in that it was the most succinct (seven pages long) and was endorsed nationally by the British Thoracic Society and supported by the Royal Colleges of Physicians and of General Practice. It was recognised at the time that the guidelines would need to be revised to take account of new developments and also in the light of experience of using them. The revision, which appears as a supplement to Thorax, has retained the stepwise approach to management of asthma but differs in two important respects. Firstly, it includes management of children and adults in the same document, recognising that most general practitioners treat all age groups and that asthma does not suddenly change as patients transfer from paediatric to adult hospital care. Secondly, the guidelines include summary or poster versions (which will also be appearing in the British Medical foumal) that reduce the information from the main document into a much more succinct form. The posters are intended to be simple and accessible reference sheets for use at the point of delivery of care, with different versions for the accident and emergency department, the wards, and general practice.

\section{Who is giving guidance?}

Although respiratory physicians set the ball rolling the present document is a multidisciplinary effort, in which paediatricians, general practitioners, and accident and emergency consultants have been active contributors. The process of production, from the initiation of background papers to the meetings at which the details evolved to the editorial revision, have been carried out independently of any pharmaceutical company support or participation. Thus the result is untarnished professional advice that we hope represents the best clinical deal for the patient.

\section{What are the guidelines?}

The guidelines provide a practical framework within which nearly all patients with asthma can be managed. They describe what is believed to be the best available asthma care. In part the advice is based on good published data but elsewhere it is based on belief about the best practice. The guidelines were deliberately not referenced extensively but do include a section at the end highlighting some areas in which further research is required. The working group unanimously reaffirmed the general principles of management, including the need for objective measurements in making the diagnosis, for the early introduction of prophylactic medication, and for long term follow up. This follow up should include peak flow measurement and a management plan tailored to, and agreed with, the individual patient. The finer details of management, however, such as the exact moment at which prophylactic treatment should be begun, remains controversial. The international version ${ }^{7}$ suggested that the threshold for starting prophylactic medication should be when inhaled bronchodilators are needed more often than two to three times weekly, whereas the revised British version suggests that it should be started if a patient is needing a bronchodilator more than once a day. For this and several other specific details evidence from trials is lacking and the guidelines represent what is believed on the basis of clinical experience to be the best course of action. While recognising the value of clinical experience we must be aware that the guidelines are not written on tablets of stone and might need to be changed when new data are produced. Those who produced the guidelines were aware of these limitations and deliberately avoided the use of the word protocol. "Protocol" implies a definitive method of treatment whereas "guideline" implies that there will always be patients for whom an individual approach outside the guidelines is both justifiable and desirable. For the most part such variations will be made by clinicians with particular understanding and experience of asthma.

\section{For whom?}

Patients with asthma not only are numerous but may receive care from many different sources, including the general practitioner, school doctor, paediatrician, respiratory physician, and accident and emergency doctor. The care may not only come from a doctor - nurses and other health care professionals have important roles both in hospital and in the community. Little disturbs patients more than receiving different advice about the same problem. The guidelines offer a framework for these various health workers to coordinate their care and indeed to decide who should do what. Such coordination is unlikely to occur by chance and each health district will need to work out its own detailed pattern of care. This will and should include the health authority "purchasers". Health authorities need to be sure that facilities exist within which the guidelines can be implemented for the substantial numbers of patients with asthma for whom they need to provide. This includes ensuring that there is someone in each district coordinating the service and that appropriate expertise for managing both adults and children is available both in the community and in the hospital. Some new medical and nursing posts will be needed and there may be fears that asthma care will be very expensive. The General Practioners In Asthma Group, however, has shown that while the cost of prescribing asthma drugs efficiently is high the overall prescribing cost of their practices is no higher than average, suggesting that appropriate care may not be more expensive. ${ }^{10}$ The national asthma audit showed that over $60 \%$ of admissions for asthma were readmissions and that $12.5 \%$ 
were readmissions within eight weeks. ${ }^{11}$ These are not reassuring figures for the patient but do offer potential cost savings if admission rates can be reduced.

\section{Where to?}

The act of producing and publishing guidelines does not change anything. For many doctors even the relatively concise 1990 UK guidelines remained as an article to read "some time". A national audit of asthma care in 36 hospitals compared care before and one year after the publication of the guidelines. Remarkably little changed in the year (M G Pearson, personal communication). The same deficiencies were still present one year later despite the guidelines and were most striking for care provided by general rather than respiratory physicians. It is much easier to produce a strategy than to implement it. The summary poster versions are a step towards getting the information to where the action occurs. The problems are not entirely medical, however. If asthma care is really to improve there will have to be a concerted and persistent thrust from an alliance of health purchasers and providers to coordinate hospital and community management in each locality. Many of the deaths from asthma are potentially preventable ${ }^{12}$ and so is much of the morbidity. As asthma is so common this becomes a major issue of "health gain". Investments in hospital and community liaison staff, in training health care professionals, and in developing educational packages that allow the patients to share actively in their own care are likely to have excellent long term returns. We owe it to our patients to see that the initiatives within the asthma guidelines are carried through into routine clinical practice, and we must be prepared to work with and through administrators and other health care professionals to ensure that end. The challenge now is to make the guidelines work.

\section{MICHAEL G PEARSON}

Fazakerley Hospital,
Liverpool L9 $7 A L$

Reprint requests to: Dr M G Pearson

1 Anonymous. Occurrence and cost of asthma. Worthing: Cambridge Medical Publications, 1990.

2 Woolcock A, Rubinfield AR, Seale JP, Landau LI, Antic R, Mitchell C, et al. Asthma management plan 1989. Med f Aust 1989;151:650-3.

3 Hargreave FE, Dolovich J, Newhouse JT. The assessment and treatment of asthma: a conference report. $\mathcal{F}$ Allergy Clin Immunol 1990;85: 1098-111.

4 Guidelines for the management of asthma in adults. 1-Chronic persisten asthma. Statement by the British Thoracic Society, Research Unit of the Royal College of Physicians of London, King's Fund Centre, National Asthma Campaign. BMF 1990;301:651-3.

5 Guidelines for the management of asthma in adults. 2-Acute severe asthma. Statement by the British Thoracic Society, Research Unit of the ma. Statement by the British Thoracic Society, Research Unit of the
Royal College of Physicians of London, King's Fund Centre, National Asthma Campaign. BMY 1990;301:797-800.

6 Warner JO, Gotz M, Landau LI, Levison H, Milner AD, Pederson S, et al. Management of asthma: a consensus statement. Arch Dis Child 1989;64:1065-79.

7 International paediatric asthma consensus group. Asthma: a follow-up statement. Arch Dis Child 1992;67:240-8.

8 Guidelines for the diagnosis and management of asthma. Bethesda Maryland: US Department of Health and Human Services, 1991.

9 International consensus report on the diagnosis and management of asthma. Clin and Experimental Allergy 1992;22(suppl): 1-72.

10 Jones $\mathrm{K}$ and the General Practioners in Asthma Group. Impact of an interest in asthma on prescribing costs in general practice Quality in Health Care 1992;1:110-3.

11 Pearson MG, Ryland I, Rudolph M, Harrison BDW. Discharge and follow-up of acute asthma [abstract]. Thorax 1992;47:209P.

12 British Thoracic Association. Deaths from asthma in two regions of England. BMF 1982;285:1251-5. 